Check for updates

Cite this: Phys. Chem. Chem. Phys., 2017, 19, 26158

Received 20th July 2017,

Accepted 5th September 2017

DOI: $10.1039 / \mathrm{c} 7 \mathrm{cp} 04890 \mathrm{~d}$

rsc.li/pccp

\section{Microscopic rigidity and heterogeneity of ionic liquids probed by stochastic molecular librations of the dissolved nitroxides $\uparrow$}

\author{
Mikhail Yu. Ivanov, ${ }^{a b}$ Olesya A. Krumkacheva, ${ }^{a b}$ Sergei A. Dzuba ${ }^{b c}$ and \\ Matvey V. Fedin (D) *ab
}

\begin{abstract}
Microscopic molecular organization and heterogeneities in ionic liquids (ILs) are of significant fundamental and applied interest. Although many theoretical studies have been dedicated to this topic, the development of experimental methods for studying such heterogeneities is still in demand. In this work we propose a new approach for the characterization of microscopic rigidity and heterogeneities in ILS using stochastic librations (small angle motions) of the nitroxide radicals as a probe. Stable nitroxides are dissolved in ILs, which are then shock-frozen and investigated using pulse Electron Paramagnetic Resonance (EPR) at variable temperatures. Stochastic molecular librations of nitroxides depend on local rigidity of a medium and manifest themselves in different electron dephasing times across the EPR spectrum. The use of advanced spiro-cyclohexane-substituted nitroxides allows implementation of this approach at temperatures up to $\sim 150 \mathrm{~K}$ and above. Remarkably, we have found that librational motions in ILs arise at much lower temperatures $\left(\sim 75 \mathrm{~K}\right.$ for $\left[\mathrm{Bmim}_{\mathrm{B}} \mathrm{BF}_{4},[\mathrm{Bmim}] \mathrm{PF}_{6},\left[\mathrm{C}_{10} \mathrm{mim}\right] \mathrm{BF}_{4}\right)$ compared to common organic solvents. This can be rationalized by smaller local rigidity of the medium in ILs, most likely due to localization of nitroxides in low-density heterogeneities formed by non-polar alkyl chains. The structure, size, solubility and other properties of nitroxides are adjustable for particular tasks, therefore the proposed approach can potentially be implemented to probe the rigidity and heterogeneities of any ILs, thus providing vital insights into their molecular-scale self-organization.
\end{abstract}

\section{Introduction}

Ionic liquids (ILs) have drawn enormous attention over the past few years in the field of green chemistry. ${ }^{1-4}$ They are broadly considered as alternatives to the common organic solvents, being applicable in industrial processes, and the same time considerably less toxic and therefore bio- and environmentallyfriendly. Being in essence the melting salts, they are comprised of cations and anions which do not recombine into a neutral salt due to steric factors. Apart from the use of ILs as advanced solvents, their unusual physico-chemical properties ${ }^{5-8}$ are intensively investigated with potential to find new areas of their implementation, e.g. photolysis, redox and polymerization reactions, catalysis, and biomedicine. $^{9-12}$ In this regard, most appealing specifics of ILs is their nano-to-microscale self-organization, leading to the formation

\footnotetext{
${ }^{a}$ International Tomography Center SB RAS, 630090, Novosibirsk, Russia. E-mail:mfedin@tomo.nsc.ru

${ }^{b}$ Novosibirsk State University, 630090, Novosibirsk, Russia

${ }^{c}$ Voevodsky Institute of Chemical Kinetics and Combustion SB RAS, 630090, Novosibirsk, Russia

$\dagger$ Electronic supplementary information (ESI) available. See DOI: 10.1039/ c7cp04890d
}

of heterogeneities. ${ }^{4,13-25}$ Such heterogeneities can potentially be used to alter the reaction pathways and yields, similar to the effect of micelles or other molecular cages. However, at the moment this is still a mostly unexplored field.

Over the past decade, a lot of theoretical investigations have been conducted to study the effects of molecular self-organization in ILs. ${ }^{14-16}$ Many studies demonstrated that charged cationic head groups and anions can aggregate in polar nanodomains, whereas hydrophobic alkyl chains of cations comprise nonpolar nanodomains in some way resembling micelles. The size of such nonpolar domains depends on the length of the corresponding alkyl chain of the cation and is larger for longer chains. Later on, experimental evidence for nanostructuring in ILs began to appear. Methods such as X-ray diffraction/scattering, ${ }^{16-18,22}$ neutron diffraction, ${ }^{26}$ quasi-elastic neutron scattering ${ }^{27}$ were intensively used. Recently, magnetic resonance methods ${ }^{20,28,29}$ and electron microscopy ${ }^{21}$ were also involved in the study of heterogeneities and nanostructuring in ILs. However, still the number of experimental approaches to detect and characterize the heterogeneities formed in ILs is relatively small.

Electron paramagnetic resonance (EPR) of spin probes is one of the potent tools used to access this information. It is well 
established that the continuous wave (CW) EPR spectrum of nitroxide radicals in solution is very sensitive to the radical's motion. ${ }^{30}$ Theoretical analysis of the experimental CW EPR spectra allows obtaining rotational correlation times and local viscosity, as well as information on anisotropy of rotation, ${ }^{30}$ if it is present e.g. due to some steric hindrances. CW and pulse EPR of nitroxide spin probes is extensively used in biochemical applications, ${ }^{31-33}$ and has also been applied in a series of works to reveal peculiarities of ILs. ${ }^{34-44}$ In particular, it was demonstrated that microscopic viscosity experienced by radicals might be considerably different from the corresponding macroscopic value. $^{28,34}$ The crystallization, glass formation and softening of ILs vs. temperature were also addressed. ${ }^{42}$ In some cases, high-field pulse EPR was applied to characterize the ways of nitroxide dissolvation in ILs and IL-water mixtures. ${ }^{43}$ However, the information provided by all these methods on nanoscale heterogeneities is still not sufficient. Recently we demonstrated another approach based on time-resolved (TR) EPR of photoexcited triplet molecules. ${ }^{28,29}$ We have shown that thorough analysis of TR EPR data indicates a specific behavior in ILs compared to common organic solvents, which implies two types of microenvironments around a spin probe. Although this approach appears to be more sensitive to detect the presence of heterogeneities compared to CW EPR, there is still a need to enhance information obtainable on the dimensions and physical properties of such heterogeneities.

In the present work we propose another approach which can be fruitfully used to characterize nanostructuring in ILs, and which, to the best of our knowledge, has never been applied for this task so far. It is based on the sensitivity of electron spin echo (ESE) to the stochastic molecular librations (small angle tumbling) that occur on the nanosecond time scale. In all cases the librations manifest themselves depending on electron spin dephasing times ( $T_{\mathrm{m}}$, phase memory times) vs. field position within the spectrum of nitroxide. The comparison of $T_{\mathrm{m}}$ measured at different field positions allows extracting the motional parameter $\left\langle\alpha^{2}\right\rangle \tau_{\mathrm{c}}$, where $\left\langle\alpha^{2}\right\rangle$ is the mean squared angular amplitude of motion and $\tau_{\mathrm{c}}$ is the corresponding correlation time. The lowest $\left\langle\alpha^{2}\right\rangle$ value that can be measured with this approach was estimated previously as $10^{-5} \operatorname{rad}^{2} .^{45}$ It was supposed that stochastic molecular librations observed by EPR in organic solvents (glycerol and $o$-terphenyl) and in membranes have the same nature as the large-amplitude anharmonic (or stochastic) atomic vibrations detected in glasses using neutron scattering and Mössbauer absorption techniques. ${ }^{46,47}$

Stochastic molecular librations of nitroxides in glasses were intensively studied using EPR and allowed revealing interesting properties of the media such as e.g. harmonic-anharmonic dynamical transitions. ${ }^{46,48-50}$ In addition, the same approaches allowed important conclusions on structural organization of some biological membrane systems. ${ }^{47,51,52}$ Below we describe the first application of the libration-based approach to characterize the local surroundings of nitroxides in neat ILs using well-known $[\mathrm{Bmim}] \mathrm{BF}_{4},[\mathrm{Bmim}] \mathrm{PF}_{6}$ and $\left[\mathrm{C}_{10} \mathrm{mim}\right] \mathrm{BF}_{4}$ as examples and several common organic solvents for comparison. We discuss the information ability and scope of applications of the proposed approach.

\section{Experimental}

The ionic liquids 1-butyl-3-methylimidazolium tetrafluoroborate ([Bmim] $\mathrm{BF}_{4}$ ), 1-butyl-3-methylimidazolium hexafluorophosphate ([Bmim] $\mathrm{PF}_{6}$ ), and 1-decyl-3-methylimidazolium tetrafluoroborate $\left(\left[\mathrm{C}_{10} \mathrm{mim}\right] \mathrm{BF}_{4}\right)$ were purchased from Sigma-Aldrich and used without additional purification (Scheme 1a).

The spiro-cyclohexane-substituted nitroxides N1 and N2 (Scheme 1b) were kindly provided by Dr Igor Kirilyuk, NIOCh SB RAS. They were dissolved in the corresponding IL in concentrations of $\approx 1 \mathrm{mM}$ and exposed to 3-5 freeze-pump-thaw cycles. Next, the solution in the quartz tube was evacuated $\left(10^{-2}\right.$ Torr pressure) with simultaneous heating at $75{ }^{\circ} \mathrm{C}$ for 3 hours to reduce the amount of remaining water in the IL and to eliminate the remaining oxygen. Finally, the tube was sealed off under vacuum.

EPR measurements were performed using a commercial Bruker Elexsys E580 spectrometer at the X-band. The spectrometer was equipped with an Oxford Instruments temperature control system (4-300 K). The echo-detected EPR spectra and phase memory times were recorded using the standard twopulse echo sequence with pulse lengths being typically $100 \mathrm{~ns}$ for $\pi$ and $50 \mathrm{~ns}$ for $\pi / 2$.

\section{Results and discussion}

In this work we use a set of three representative ILs (Scheme 1a). $[\mathrm{Bmim}] \mathrm{BF}_{4}$ and $[\mathrm{Bmim}] \mathrm{PF}_{6}$ are among the most used and widespread ILs. Since they have the same cation [Bmim] but different anions $\mathrm{BF}_{4}$ and $\mathrm{PF}_{6}$, the comparison of trends obtained in these two ILs addresses the role of anions in the observed phenomena. The third IL $\left[\mathrm{C}_{10} \mathrm{mim}\right] \mathrm{BF}_{4}$ has the same anion as $[\mathrm{Bmim}] \mathrm{BF}_{4}$, but a different cation, and thus can

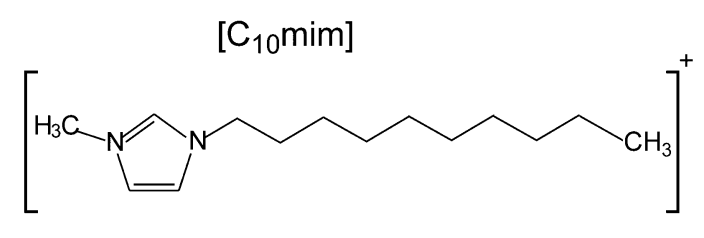

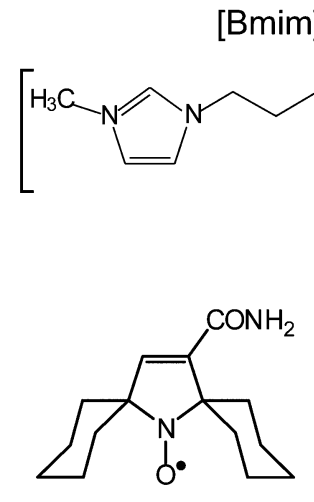

N1

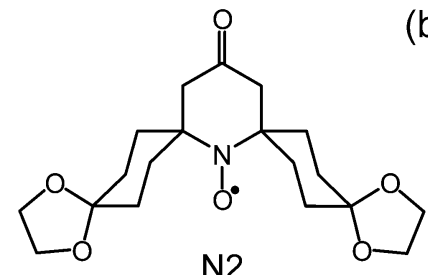

N2

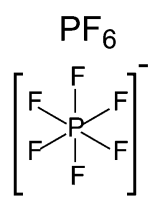

(b)
Scheme 1 (a) Chemical structure of cations and anions of the studied ILS $[B$ mim $] \mathrm{BF}_{4},\left[\mathrm{Bmim}_{\mathrm{P}} \mathrm{PF}_{6},\left[\mathrm{C}_{10} \mathrm{mim}_{\mathrm{BF}}\right.\right.$. (b) The structure of nitroxide probes used. 
be used to elucidate the role of the cation. The same set of three ILs was used in our previous work to benchmark information capabilities of time-resolved EPR, which indicated the occurrence of heterogeneities in these ILs. ${ }^{28}$ Therefore, the same three ILs were used here as the test grounds for a new approach.

Most common limitation of pulse EPR techniques is rooted in short phase memory times $\left(T_{\mathrm{m}}\right)$ of studied paramagnetic centers precluding the observation of electron spin echo (ESE). Nitroxides are the most widespread spin probes in EPR, however $T_{\mathrm{m}}$ of most of the nitroxides drastically shortens above $\sim 80 \mathrm{~K}$ due to the onset of methyl group rotation adjacent to the NO moiety. Recently this drawback was remedied by developing the nitroxides with spiro-cyclohexane fragments replacing the $\mathrm{CH}_{3}$ groups (Scheme 1b). ${ }^{55-57}$ For such improved probes rapid acceleration of $T_{\mathrm{m}}$ begins at considerably higher temperatures $\sim 135 \mathrm{~K}$, therefore they can be used for pulse EPR studies in a much broader temperature range. In this work we used spin probe $\mathrm{N} 1$ in all experiments, whereas more bulky nitroxide N2 was used only once to verify the influence of the probe's size.

Solutions of nitroxides in ILs were shock-frozen (77 K) and then investigated at variable temperatures. Although the local structure in the liquid and frozen state of ILs might be slightly different, we assume that key characteristics such as heterogeneity, segregation of polar/non-polar regions, localization of nitroxides etc. are captured by shock-freezing, similar to the same paradigm broadly used in biological pulse EPR.

Fig. 1 shows typical two-pulse echo-detected (ED) EPR spectra measured in $[\mathrm{Bmim}] \mathrm{PF}_{6}$ at $50 \mathrm{~K}$ and $120 \mathrm{~K}$ for different pulse separation times $\tau$ (Hahn's sequence $\pi / 2-\tau-\pi$ - $\tau$-echo). In order to investigate the evolution of the spectrum shape $v s$. $\tau$, all spectra were normalized to the maximum. One observes that at $50 \mathrm{~K}$ the shape of the spectrum does not significantly change with $\tau$, whereas at $120 \mathrm{~K}$ the relative intensity of the high-field and lowfield shoulders strongly drops at long $\tau$ delays. The librations induce stronger stochastic fields and thus most pronounced shortening of $T_{\mathrm{m}}$ in most "anisotropic" spectral positions (far from canonical orientations), therefore mainly canonical orientations contribute to the ED EPR spectrum at long times $\tau$.

Insets of Fig. 1 demonstrate typical ESE decays measured for nitroxide $\mathrm{N} 1$ in $\left[\mathrm{Bmim}^{-} \mathrm{PF}_{6}\right.$ at central and high-field spectral positions in the absence $(50 \mathrm{~K})$ or presence $(120 \mathrm{~K})$ of stochastic molecular librations. As was shown previously, the librations can be essentially described by two parameters: angular amplitude of motion $\alpha$ and its characteristic correlation time $\tau_{\mathrm{c}}$; however, experimentally one obtains their combination $\left\langle\alpha^{2}\right\rangle \tau_{\mathrm{c}}$, where $\left\langle\alpha^{2}\right\rangle$ is the mean squared angular amplitude. ${ }^{47}$ This motional parameter $\left\langle\alpha^{2}\right\rangle \tau_{\text {c }}$ can be straightforwardly obtained by taking the difference $(\Delta W)$ of $T_{\mathrm{m}}{ }^{-1}$ values measured at two spectral positions shown by arrows in Fig. 1. Previous work has shown that $\Delta W$ can be expressed as $\Delta W \approx 9 \times 10^{16} \mathrm{~s}^{-2} \cdot\left\langle\alpha^{2}\right\rangle \tau_{\mathrm{c}} \operatorname{rad}^{2} \mathrm{s.}^{47}$

Fig. 2 shows the obtained temperature dependencies of the

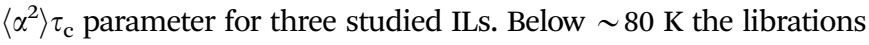
in ILs vanish, and at $50 \mathrm{~K}$ they are clearly absent. The dependencies can be approximated by linear fits at $T=80-150 \mathrm{~K}$ for $[\mathrm{Bmim}] \mathrm{BF}_{4}$ and $[\mathrm{Bmim}] \mathrm{PF}_{6}$, and at $T=80-140 \mathrm{~K}$ for $\left[\mathrm{C}_{10} \mathrm{mim}\right] \mathrm{BF}_{4}$ (vide infra). Such a linear behavior is typical for previous studies of stochastic
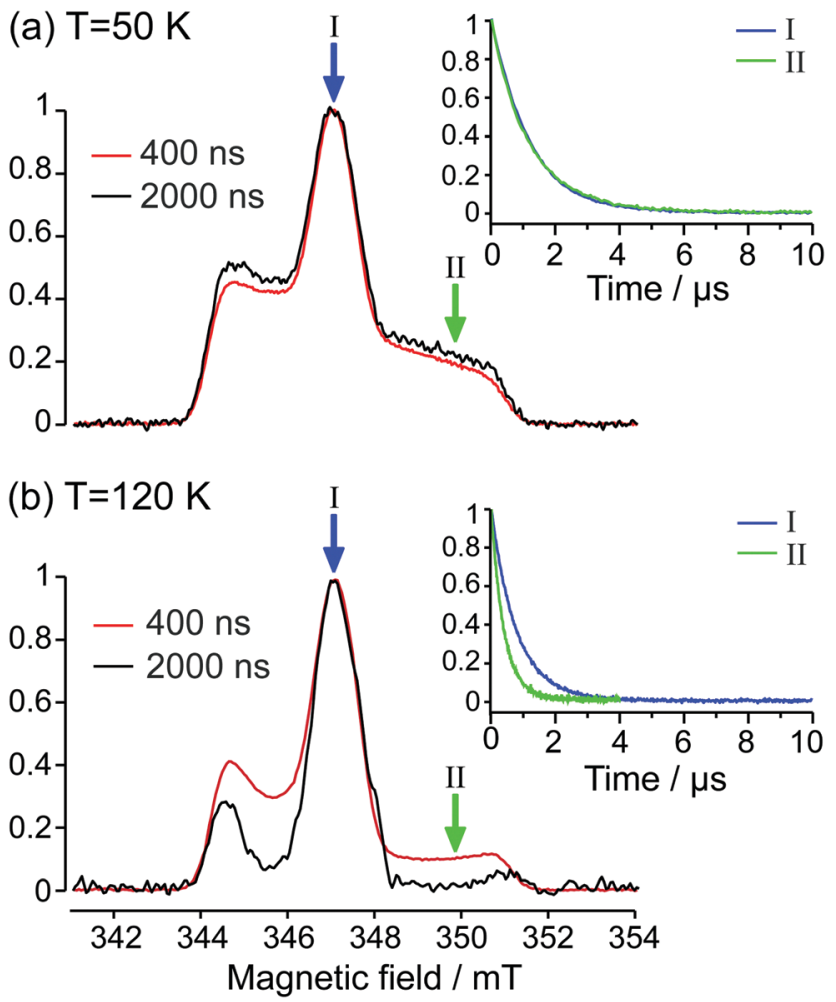

Fig. 1 Normalized two-pulse ED EPR spectra of N1 measured in [Bmim]PF 6 at $50 \mathrm{~K}(\mathrm{a})$ and $120 \mathrm{~K}$ (b) for different pulse separation times $\tau$ (400 and 2000 ns, as indicated) in Hahn's sequence. Insets: ESE decays measured at central (blue arrow, I) and high-field (green arrow, II) spectral positions.

molecular librations in organic glasses and biological membranes. ${ }^{4-54}$ At the temperature range $80-150 \mathrm{~K}$ the librations systematically appear to be more intensive in ILs compared to common organic solvents. Here we used glycerol, o-terphenyl and squalane as representatives of common solvents because they preserve the glass state at a very large range of temperatures,

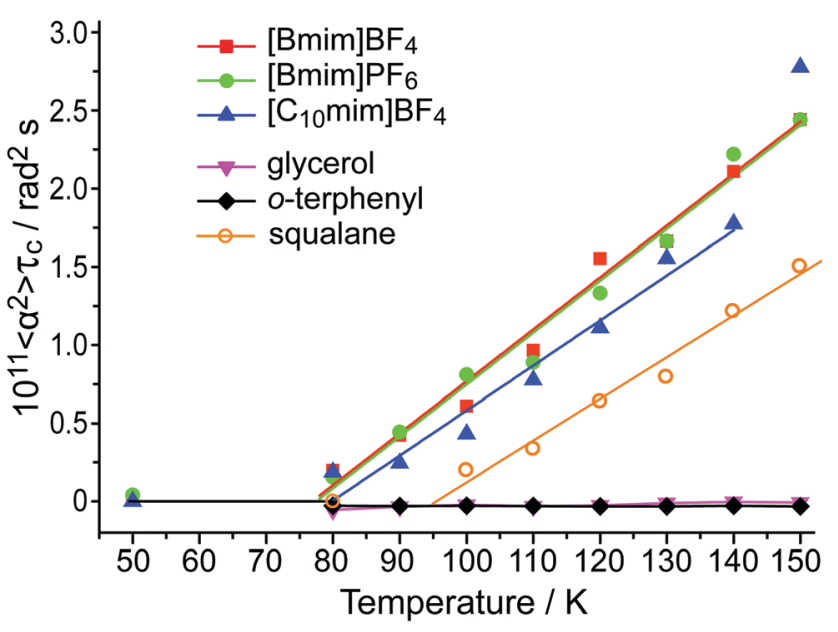

Fig. 2 Temperature dependence of the motional parameter $\left\langle\alpha^{2}\right\rangle \tau_{\mathrm{c}}$ for N1 in ILs and organic solvents (indicated in the plot). The solid lines show linear fits (see in the text). 
whereas many other organic solvents (e.g. toluene) exhibit glass to crystal transitions at $T<150 \mathrm{~K}$. The librations of nitroxide in glycerol and $o$-terphenyl are not detectable at $T=80-150 \mathrm{~K}$. However, in less rigid glass of squalane, whose structure in some way resembles long alkyl chains of $\mathrm{C}_{10} \mathrm{mim}$, decent librations are observed at $T>100 \mathrm{~K}$, but the motional parameter $\left\langle\alpha^{2}\right\rangle \tau_{\mathrm{c}}$ is still much smaller than that in ILs at the same temperature. Since the same spin probe $\mathrm{N} 1$ was used in all cases, such a behavior clearly implies higher librational mobility of the nitroxide in ILs compared to frozen organic solvents.

This observation implies that the nitroxides in frozen ILs are localized in the regions of low rigidity, i.e. low density. Most likely, this corresponds to the location of nitroxides in nonpolar regions formed by segregated alkyl chains. In such "micelle-like" environments ${ }^{24}$ the tumbling of nitroxide must be much less restricted. Fig. 2 shows that in general temperature dependencies of $\left\langle\alpha^{2}\right\rangle \tau_{\mathrm{c}}$ are very close for all three studied ILs. The values of $\left\langle\alpha^{2}\right\rangle \tau_{\mathrm{c}} v s$. $T$ for $\left[\mathrm{Bmim}^{2}\right] \mathrm{BF}_{4}$ and $[\mathrm{Bmim}] \mathrm{PF}_{6}$ are indistinguishable from each other within experimental accuracy. However, the values of $\left\langle\alpha^{2}\right\rangle \tau_{\mathrm{c}}$ for $\left[\mathrm{C}_{10} \mathrm{mim}\right] \mathrm{BF}_{4}$ are systematically smaller compared to two other ILs in the temperature range between 80 and $130 \mathrm{~K}$. Our previous work has demonstrated that at higher temperatures all three studied ILs exhibit a biphasic behavior with gradual transition to a melted state, which begins at $T \sim 140 \mathrm{~K}$ for $\left[\mathrm{C}_{10} \mathrm{mim}\right] \mathrm{BF}_{4}$ and at higher $T \sim 170 \mathrm{~K}$ for $\left[\mathrm{Bmim}^{\mathrm{T}}\right] \mathrm{BF}_{4}$ and $[\mathrm{Bmim}] \mathrm{PF}_{6}{ }^{28}{ }^{28}$ We believe that this is the reason why $\left\langle\alpha^{2}\right\rangle \tau_{\mathrm{c}}$ dependencies of $\left[\mathrm{C}_{10} \mathrm{mim}\right] \mathrm{BF}_{4}$ and two other ILs intersect at $\sim 135 \mathrm{~K}$; this also is the reason why in the present work we do not consider stochastic molecular librations at $T>150 \mathrm{~K}$. Thus, similarity of $\left\langle\alpha^{2}\right\rangle \tau_{\mathrm{c}}$ dependencies for $[\mathrm{Bmim}] \mathrm{BF}_{4}$ and $[\mathrm{Bmim}] \mathrm{PF}_{6}$ shows that the impact of anions on librations of nitroxide is minor, whereas the comparison with $\left[\mathrm{C}_{10} \mathrm{mim}\right] \mathrm{BF}_{4}$ at $T=80-130 \mathrm{~K}$ indicates some decent influence of the cation. Once again, this agrees with the hypothesis of the nitroxide preferable location in non-polar regions of alkyl chains.

It is particularly interesting to compare the results obtained in ILs and in squalane. Among three studied ILs, the $\left\langle\alpha^{2}\right\rangle \tau_{\mathrm{c}}$ dependence in $\left[\mathrm{C}_{10} \mathrm{mim}\right] \mathrm{BF}_{4}$ is closer to that in squalane, but still the librations in $\left[\mathrm{C}_{10} \mathrm{mim}\right] \mathrm{BF}_{4}$ are much more intensive compared to squalane. This clearly indicates that more intensive librations in ILs are caused not by merely a localization of nitroxide within alkyl chains, but by the occurrence of non-polar heterogeneities less dense than the "pure-chain" squalane. One reasonably assumes that the strongest manifestation of heterogeneities should occur when the dimensions of polar and nonpolar regions are comparable. This is about to be fulfilled for $[\mathrm{Bmim}] \mathrm{BF}_{4}$ and $[\mathrm{Bmim}] \mathrm{PF}_{6}$, and should gradually vanish upon increasing of the alkyl chain length to approach the homogeneous properties of a common solvent.

Fig. 3 shows ESE decays for N1 in ILs and in o-terphenyl measured at the global maximum of the EPR spectrum (position I in Fig. 1). The ESE decay for N1 in the organic solvent $o$-terphenyl is mainly determined by the electron-nuclear spin-spin interactions between $\mathrm{N} 1$ and surrounding matrix protons (nuclear spin diffusion mechanism), and is described by expression

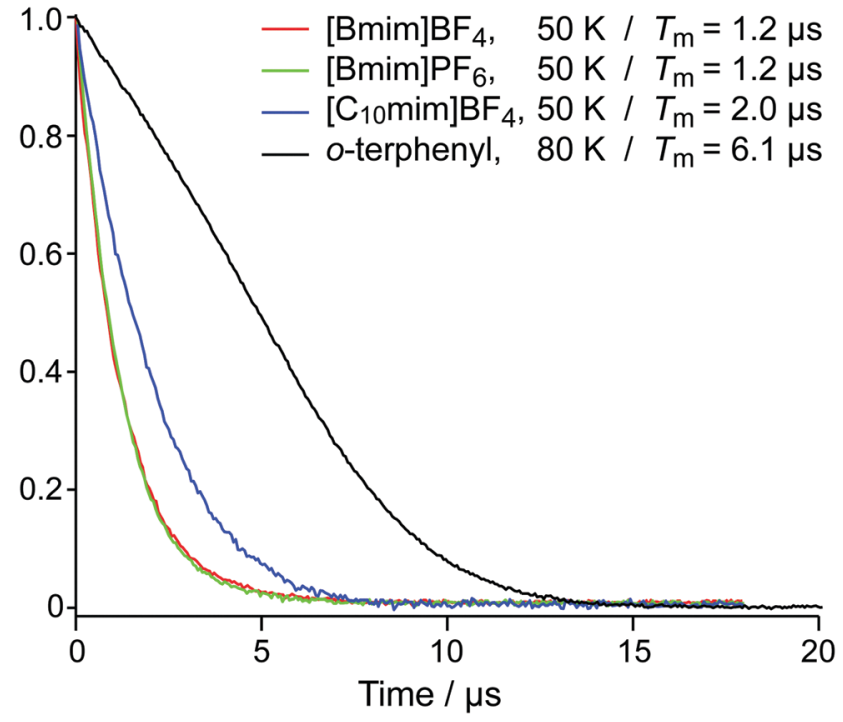

Fig. 3 ESE decays for N1 in ILs and in o-terphenyl (solvent and temperatures are indicated) measured at the global maximum of the EPR spectrum (position I of Fig. 1). The $T_{m}$ values correspond to the time of signal decrease by a factor of $e$.

$I=I_{0} \exp \left(-\left(t / T_{\mathrm{m}}\right)\right)^{n}$, where $n>1 .^{58}$ At the same time, ESE decays in all three ILs are described by the monoexponential function $(n=1)$ with $T_{\mathrm{m}}$ values being significantly smaller compared to $o$-terphenyl. This indicates that the additional relaxation mechanisms contribute to $T_{\mathrm{m}}$ values in ILs. Most likely, the shorter $T_{\mathrm{m}}$ values in ILs are determined by additional interactions between unpaired electrons of the nitroxide with the charged moieties of ILs modulated by matrix vibrations. Notably, the $T_{\mathrm{m}}$ values in $[\mathrm{Bmim}] \mathrm{BF}_{4}$ and $[\mathrm{Bmim}] \mathrm{PF}_{6}$ are very similar, whereas $T_{\mathrm{m}}$ in $\left[\mathrm{C}_{10} \mathrm{mim}\right] \mathrm{BF}_{4}$ is significantly different, being closer to that of $o$-terphenyl. This again agrees well with the hypothesis that the nitroxides are preferably localized within the bundle of long $\left(\mathrm{C}_{10}\right)$ alkyl chains. In such a case the additional relaxation mechanism induced by the charged moieties should be less efficient in $\left[\mathrm{C}_{10} \mathrm{mim}\right] \mathrm{BF}_{4}$ compared to [Bmim]-based ILs, because the nitroxide is better shielded or more distant from the charges due to the longer alkyl chains. As a result, we observe that the $T_{\mathrm{m}}$ character in $\left[\mathrm{C}_{10} \mathrm{mim}\right] \mathrm{BF}_{4}$ is closer to that in common organic solvents.

The characteristic temperature range where stochastic molecular librations in ILs are active agrees well with the previous observations for [Bmim] $\mathrm{PF}_{6}$ using quasielastic neutron scattering experiments (QENS). ${ }^{27}$ The authors characterized motional dynamics in ILs considering three temperature regimes. Below $100 \mathrm{~K}$ only methyl group rotation occurs, whereas at $T \sim 100-200 \mathrm{~K}$ the butyl side chains overcome the activation energy and relax into various conformations, and above $T \sim 200 \mathrm{~K}$ (glass transition temperature) the onset of diffusive motion is observed. Thus, the growth of the motional parameter $\left\langle\alpha^{2}\right\rangle \tau_{\mathrm{c}}$ found by us at $T>80 \mathrm{~K}$ is consistent with an increased flexibility of alkyl chains. Once again, this agrees well with the hypothesis of preferred localization of nitroxide in non-polar regions of ILs within the alkyl chains. 
We have also tested the influence of the probe size by performing a few measurements in $\left[\mathrm{Bmim}^{-} \mathrm{PF}_{6}\right.$ using more bulky nitroxide N2 (ESI $\dagger)$. If the librational motion of the NO group was influenced to some extent by the overall mobility of the nitroxide, one would anticipate the influence of the radical size on $\left\langle\alpha^{2}\right\rangle \tau_{\mathrm{c}}$ vs. T. However, as is shown in Fig. S1 ESI, $\dagger$ the differences between $\left\langle\alpha^{2}\right\rangle \tau_{\mathrm{c}}$ dependencies obtained for N1 and $\mathrm{N} 2$ in the same IL are negligible. This means that the character of librations is local with respect to the NO group, and is rather determined by the interaction with the surrounding matrix and its properties. Note that the approach presented in this work can also be implemented using more common tetramethylsubstituted nitroxides, but in the narrower temperature range (see above). In addition, the nitroxides can be functionalized in order to favor their preferred locations in polar/non-polar regions of ILs, as was shown e.g. in ref. 43, and in this way to enhance the obtained information on nanostructures formed.

Finally, it is outstanding how similar are the $\left\langle\alpha^{2}\right\rangle \tau_{\mathrm{c}}$ vs. $T$ dependencies obtained for three studied ILs here (Fig. 2) to those obtained previously for biological membranes. ${ }^{45,54}$ For instance, there are both qualitative and quantitative similarities in $\left\langle\alpha^{2}\right\rangle \tau_{\mathrm{c}} v s$. $T$ obtained for the nitroxides in ILs and nitroxides located in the shell of bilayers consisting of unsaturated lipid 1-palmitoyl-2-oleoyl-sn-glycero-3-phosphocholine (POPC) (see Fig. 5 of ref. 45). The onset of stochastic molecular librations in studied ILs occurs at lower temperatures compared to POPC ( $\sim 75 \mathrm{~K} v s . \sim 90 \mathrm{~K}$, respectively), however the slopes of the dependencies and the values at $T \sim 150 \mathrm{~K}$ are reasonably close $\left(\sim 2.5 \operatorname{rad}^{2} \mathrm{~s}\right)$. Since the occurrence of low-density domains in membranes and their loose packing are well established, we emphasize that the observed similarities with ILs strongly assume the formation of analogous domains. This also highlights the resemblance of local surroundings experienced by small molecules in these two types of media, which can be used in future e.g. for biomedical applications of ILs.

\section{Conclusions}

In this work we have proposed and verified a new experimental approach for the characterization of local microscopic-level rigidity and heterogeneities in ILs. Using three representative ILS $[\mathrm{Bmim}] \mathrm{BF}_{4},[\mathrm{Bmim}] \mathrm{PF}_{6}$ and $\left[\mathrm{C}_{10}\right.$ mim $] \mathrm{BF}_{4}$ we have shown that the stochastic molecular librations of dissolved nitroxides acting as a probe provide evidence for much smaller rigidity in non-polar areas of ILs compared to common organic solvents. The motional parameter of librations $\left\langle\alpha^{2}\right\rangle \tau_{\mathrm{c}}$ in studied ILs at $T=80-150 \mathrm{~K}$ is one to two orders of magnitude larger compared to common organic solvents such as glycerol and $o$-terphenyl, which is most plausibly explained by localization of nitroxides in the low-density regions of segregated non-polar alkyl chains. Although we have demonstrated a new approach only for three selected ILs, we note that the structure, size, solubility and other properties of nitroxides are adjustable for particular tasks, therefore the proposed approach can potentially be implemented to probe the rigidity and heterogeneities of any ILs in order to elucidate their molecular-scale self-organization.
Interestingly, the observed onset of low temperature stochastic molecular librations in ILs resembles previous observations made in biological membranes. ${ }^{45,54}$ Membranes are certainly heterogeneous systems, where the librational mobility of nitroxides can be more or less restricted depending on localization. Similar microscopic rigidity assumes that perhaps in some cases frozen ILs can be used to model biologically-relevant media. Whether this idea will prove feasible or not, the first demonstration of the libration-based approach to study rigidity and heterogeneity of ILs certainly expands this informative methodology for the new class of systems and boosts its applications in general.

\section{Conflicts of interest}

There are no conflicts to declare.

\section{Acknowledgements}

This work was supported by the Russian Science Foundation (No. 14-13-00826). We are indebted to Dr Igor Kirilyuk (NIOCh SB RAS) for providing us with the nitroxide spin probes.

\section{References}

1 T. Welton, Chem. Rev., 1999, 99, 2071.

2 J. P. Hallett and T. Welton, Chem. Rev., 2011, 111, 3508.

3 J. Dupont, R. F. de Souza and P. A. Suarez, Chem. Rev., 2002, $102,3667$.

4 R. D. Rogers and G. A. Voth, Acc. Chem. Res., 2007, 40, 1077.

5 L. Crowhurst, P. R. Mawdsley, J. M. Perez-Arlandis, P. A. Salter and T. Welton, Phys. Chem. Chem. Phys., 2003, 5, 2790-2794.

6 M. Deetlefs, K. R. Seddon and M. Shara, Phys. Chem. Chem. Phys., 2006, 8, 642-649.

7 J. Dupont and P. A. Z. Suarez, Phys. Chem. Chem. Phys., 2006, 8, 2441-2452.

8 K. Fumino, T. Peppel, M. Geppert-Rybczynska, D. H. Zaitsau, J. K. Lehmann, S. P. Verevkin, M. Kockerling and R. Ludwig, Phys. Chem. Chem. Phys., 2011, 13, 14064-14075.

9 M. Alvaro, B. Ferrer, H. Garcia and M. Narayana, Chem. Phys. Lett., 2002, 362, 435.

10 V. I. Parvulescu and C. Hardacre, Chem. Rev., 2007, 107, 2615.

11 I. A. Shkrob, T. W. Marin, R. A. Crowell and J. F. Wishart, J. Phys. Chem. A, 2013, 117, 5742.

12 K. S. Egorova, E. G. Gordeev and V. P. Ananikov, Chem. Rev., 2017, 117, 7132.

13 T. L. Greaves and C. J. Drummond, Chem. Soc. Rev., 2013, 42, 1096.

14 Y. Wang and G. A. Voth, J. Am. Chem. Soc., 2005, 127, 12192. 15 J. Lopes and A. A. H. Padua, J. Phys. Chem. B, 2006, 110, 3330. 16 A. Triolo, O. Russina, H.-J. Bleif and E. Di Cola, J. Phys. Chem. B, 2007, 111, 4641.

17 O. Russina, A. Triolo, L. Gontrani and R. Caminiti, J. Phys. Chem. Lett., 2012, 3, 27. 
18 O. Russina and A. Triolo, Faraday Discuss., 2012, 154, 97.

19 Y. Ji, R. Shi, Y. Wang and G. Saielli, J. Phys. Chem. B, 2013, 117, 1104.

20 M. M. Seitkalieva, A. A. Grachev, K. S. Egorova and V. P. Ananikov, Tetrahedron, 2014, 70, 6075.

21 A. S. Kashin, K. I. Galkin, E. A. Khokhlova and V. P. Ananikov, Angew. Chem., Int. Ed., 2016, 55, 2161.

22 C. S. Santos, H. V. R. Annapureddy, N. S. Murthy, H. K. Kashyap, E. W. Castner, Jr. and C. J. Margulis, J. Chem. Phys., 2011, 134, 064501.

23 A. M. Funston, T. A. Fadeeva, J. F. Wishart and E. W. Castner, Jr., J. Phys. Chem. B, 2007, 111, 4963.

24 H. V. R. Annapureddy, H. K. Kashyap, P. M. De Biase and C. J. Margulis, J. Phys. Chem. B, 2010, 114, 16838.

25 V. Hoffmann, A. Lahiri, N. Borisenko, T. Carstens, G. Pulletikurthi, A. Borodin, R. Atkin and F. Endres, Phys. Chem. Chem. Phys., 2017, 19, 54-58.

26 R. Hayes, S. Imberti, G. G. Warr and R. Atkin, Phys. Chem. Chem. Phys., 2011, 13, 13544.

27 A. Triolo, A. Mandanici, O. Russina, V. Rodriguez-Mora, M. Cutroni, C. Hardacre, M. Nieuwenhuyzen, H.-J. Bleif, L. Keller and M. A. Ramos, J. Phys. Chem. B, 2006, 110, 21357.

28 M. Yu. Ivanov, S. L. Veber, S. A. Prikhod'ko, N. Yu. Adonin, E. G. Bagryanskaya and M. V. Fedin, J. Phys. Chem. B, 2015, 119, 13440.

29 M. Yu. Ivanov, S. A. Prikhod'ko, N. Yu. Adonin, E. G. Bagryanskaya and M. V. Fedin, Z. Phys. Chem., 2017, 231, 391.

30 G. Moro and J. H. Freed, J. Phys. Chem., 1980, 84, 2837.

31 Distance Measurements in Biological Systems by EPR, in Biological Magnetic Resonance, ed. L. J. Berliner, S. S. Eaton and G. R. Eaton, 2000, vol. 19.

32 Y. Polyhach, E. Bordignon, R. Tschaggelar, S. Gandra, A. Godt and G. Jeschke, Phys. Chem. Chem. Phys., 2012, 14, 10762.

33 K. Mobius, A. Savitsky, A. Schnegg, M. Plato and M. Fuchs, Phys. Chem. Chem. Phys., 2005, 7, 19.

34 B. Y. Mladenova, D. R. Kattnig and G. Grampp, J. Phys. Chem. B, 2011, 115, 8183.

35 B. Y. Mladenova, N. A. Chumakova, V. I. Pergushov, A. I. Kokorin, G. Grampp and D. R. Kattnig, J. Phys. Chem. B, 2012, 116, 12295.

36 M. A. M. NoeI, R. D. Allendoerfer and R. A. Osteryoung, J. Phys. Chem., 1992, 96, 2391.

37 A. Kawai, T. Hidemori and K. Shibuya, Chem. Lett., 2004, 33, 1464.
38 R. G. Evans, A. J. Wain, C. Hardacre and R. G. Compton, ChemPhysChem, 2005, 6, 1035.

39 V. Strehmel, A. Laschewsky, R. Stoesser, A. Zehl and W. Herrmann, J. Phys. Org. Chem., 2006, 19, 318.

40 V. I. Pergushov, N. A. Chumakova, M. Ya. Mel'nikov, G. Grampp and A. I. Kokorin, Dokl. Phys. Chem., 2009, 425, 69.

41 V. Strehmel, H. Rexhausen and P. Strauch, Phys. Chem. Chem. Phys., 2010, 12, 1933.

42 V. Strehmel, ChemPhysChem, 2012, 13, 1649.

43 Y. Akdogan, J. Heller, H. Zimmermann and D. Hinderberger, Phys. Chem. Chem. Phys., 2010, 12, 7874.

44 D. R. Kattnig, Y. Akdogan, C. Bauer and D. Hinderberger, Z. Phys. Chem., 2012, 226, 1363.

45 V. N. Syryamina and S. A. Dzuba, J. Phys. Chem. B, 2017, 121, 1026.

46 S. A. Dzuba, E. P. Kirilina and E. S. Salnikov, J. Chem. Phys., 2006, 125, 054502.

47 N. P. Isaev and S. A. Dzuba, J. Phys. Chem. B, 2008, 112, 13285.

48 S. A. Dzuba, Spectrochim. Acta, Part A, 2000, 56, 227.

49 S. V. Paschenko, Y. V. Toropov, S. A. Dzuba, Y. D. Tsvetkov and A. K. Vorobiev, J. Chem. Phys., 1999, 110, 8150.

50 S. A. Dzuba, Pure Appl. Chem., 1992, 64, 825.

51 D. A. Erilov, R. Bartucci, R. Guzzi, D. Marsh, S. A. Dzuba and L. Sportelli, Biophys. J., 2004, 87, 3873.

52 R. Bartucci, R. Guzzi, M. De Zotti, C. Toniolo, L. Sportelli and D. Marsh, Biophys. J., 2008, 94, 2698.

53 V. N. Syryamina, N. P. Isaev, C. Peggion, F. Formaggio, C. Toniolo, J. Raap and S. A. Dzuba, J. Phys. Chem. B, 2010, 114, 12277.

54 N. V. Surovtsev, N. V. Ivanisenko, K. Y. Kirillov and S. A. Dzuba, J. Phys. Chem. B, 2012, 116, 8139.

55 I. A. Kirilyuk, Yu. F. Polienko, O. A. Krumkacheva, R. K. Strizhakov, Yu. V. Gatilov, I. A. Grigor'ev and E. G. Bagryanskaya, J. Org. Chem., 2012, 77, 8016.

56 E. S. Babaylova, A. V. Ivanov, A. A. Malygin, M. A. Vorobjeva, A. G. Venyaminova, Y. F. Polienko, I. A. Kirilyuk, O. A. Krumkacheva, M. V. Fedin, G. G. Karpova and E. G. Bagryanskaya, Org. Biomol. Chem., 2014, 12, 3129.

57 A. A. Kuzhelev, R. K. Strizhakov, O. A. Krumkacheva, Y. F. Polienko, D. A. Morozov, G. Yu. Shevelev, D. V. Pyshnyi, I. A. Kirilyuk, M. V. Fedin and E. G. Bagryanskaya, J. Magn. Reson., 2016, 266, 1.

58 R. Dastvan, B. E. Bode, M. P. R. Karuppiah, A. Marko, S. Lyubenova, H. Schwalbe and T. F. Prisner, J. Phys. Chem. B, 2010, 114, 13507. 